\title{
Uso da análise envoltória de dados para mensuração da sustentabilidade de hotéis-fazenda em Pernambuco
}

\author{
Socio-environmental practices in the Agreste of Pernambuco farm hotels \\ under the client optics
}

\section{Enveloppement de donnés use analyse pour la mesure-farm durabilité hôtes en Pernambuco}

\author{
Uso análisis envolvente de dados para la sostenibilidad de medición hoteles-granja en \\ Pernambuco
}

\author{
Viviane Souza* \\ Andre de Souza Melo** \\ Marcos Felipe Falcão Sobral ${ }^{* *}$ \\ Marília Nunes Valença**
}

Recebido em 23/07/2016; revisado e aprovado em 06/10/2016; aceito em 31/10/2016

DOI: http:/ /dx.doi.org/10.20435/1984-042X-2017-v.18-n.1(04)

\begin{abstract}
Resumo: O presente artigo fala da eficiência na gestão da responsabilidade socioambiental nos hotéis-fazenda da Região Agreste de Pernambuco. O universo da amostra são seis hotéis-fazenda que possuem algum apelo referente à sustentabilidade em seus sites. A pesquisa caracteriza-se como exploratória, descritiva e de corte transversal. A natureza dos dados é quantitativa usando uma metodologia não paramétrica conhecida como a Análise Envoltória de Dados (DEA). Os resultados apresentam diferenças em relação à eficiência sustentável dos hotéis em análise. Entretanto há modificação de cenário quando se alteram as variáveis (inputs e outputs) da avaliação geral, alterando a eficiência relativa das unidades. A metodologia utilizada e os resultados podem orientar a aplicação dessa pesquisa em outras realidades.
\end{abstract}

Palavras-chave: sustentabilidade; hotel-fazenda; DEA.

Abstract: This article speaks about the efficient in the social and environmental responsibility's management by farm hotels in the region Agreste of Pernambuco. The universe of the sample are six farm hotels that have some appeal for sustainability on their websites. The research is characterized as exploratory, descriptive and cross-sectional. The nature of the data is quantitative using a non-parametric methodology known as the Data Envelopment Analysis (DEA). The results show differences about to sustainable efficiency of the hotels. However, there is scenery's change when change the variables (inputs and outputs) of the general evaluation by changing the relative efficiency of the units. The methodology and results can guide the application of this research in other realities.

Key words: sustainability; farm hotel; DEA.

Résumé: Cet article nous parle de l'efficace dans la gestion de la responsabilité socio-environnemental aux hotels-ferme de la Région Agreste au Pernambuco. L'univers de l'échantillon sont les six Hôtels-Ferme qui ont quelque appel en ce qui concerne la durabilité aux sites Web. La recherche est caractérisée comme étant exploratrice, descriptive et avec une coupe transversale. La nature des données est quantitative avec l'usage d'une méthodologie non-paramétrique connue comme l'Analyse d'Enveloppement Des Données (DEA). Les résultats présentent des différences par rapport à l'efficace soutenable des hôtels à être analysés. Cependant, il y a une modification du scénario quand ils sont changés les variables (inputs et outputs) de l'évaluation générale, en changeant l'efficace relative des unités. La méthodologie usée et les résultats peuvent orienter l'application de cette recherche par rapport aux autres réalités.

Mots-clés: durabilite; hotel ferme; DEA.

\footnotetext{
* Universidade de Aveiro, Portugal.

** Universidade Federal Rural de Pernambuco, Recife, Pernambuco, Brasil.
} 
Resumen: El presente artículo habla de la eficacia en la gestión de la responsabilidad socio-ambiental en los hoteles-hacienda de la Región Agreste de Pernambuco. El universo de la muestra son seis hoteles-hacienda que tienen alguna llamada de atención referente à la sustentabilidad en sus sitios webs. La investigación se caracteriza como exploratoria, descriptiva y de corte transversal. La naturaleza de los datos es cuantitativa usando una metodología no-paramétrica conocida como la Análisis Envolvente de Datos (DEA). Los resultados presentan diferencias cuanto à la eficacia sustentable de los hoteles a ser analizados. Todavía, hay una modificación de escenario cuando se cambian las variables (inputs y outputs) de la valuación general, cambiando la eficacia relativa de las unidades. La metodología utilizada y los resultados pueden orientar la aplicación de esa pesquisa en otras realidades.

Palabras clave: sustentabilidad; hotel hacienda; DEA.

\section{INTRODUÇÃO}

A adoção e implementação de práticas de turismo sustentável pela indústria hoteleira tem sido, em parte, ocasionada pela observação de que os turistas estão cada vez mais preocupados com o meio ambiente e com as condições que possuem os destinos que pretendem visitar (MENSAH; MENSAH, 2013). Esse fator origina uma pressão mercadológica para que todos os setores do turismo adotem práticas sustentáveis a fim de se adequarem a esse novo público-alvo.

O presente artigo está alinhado com as temáticas contemporâneas respeitantes ao desenvolvimento sustentável, especialmente no que se refere à gestão da Responsabilidade SócioAmbiental Empresarial (RSAE) nas empresas. Anteriormente os termos responsabilidade ambiental e social eram utilizados de forma dissociada, contudo, nos últimos anos, de acordo com Lynes e Andrachuk (2008), esse tema tem sido tratado de forma integrada, tem despertado interesse e tem sido abordado em diversos estudos: Schaefer (2004), Egri et al. (2004), Lund-Thomsem (2004), Mcintosh (2003), Rosemberg (2004), Lynes e Andrachuk (2008), Suess (2009) e Chan (2011).

O setor da hospitalidade é uma atividade de destaque no turismo e traz consigo o papel de planejar os seus objetivos e as práticas de sustentabilidade que são pensadas para os seus âmbitos específicos (social, ambiental e econômica) (SILVA, 2012). Porém suas maiores preocupações para com o meio ambiente incluem a reciclagem e gestão de resíduos, ar puro, energia e conservação da água, saúde ambiental, manutenção de licenças, tais como as licenças de construção em conformidade com a legislação, a política de compras e educação ambiental (MENSAH, 2006). Os gestores hoteleiros precisam de uma comunicação eficaz de sistemas de monitoramento para organizar seus pensamentos e ações em relação à sustentabilidade, podendo assim, destacar qualquer incompatibilidade entre objetivos sociais e ambientais do hotel e do seu desempenho sobre essas questões (ASSAF; JOSIASSEN; CVELBAR, 2012).

Nesse contexto, foi realizada uma pesquisa exploratória, descritiva e de corte transversal, em hotéis-fazenda localizados no Estado de Pernambuco. Os dados coletados foram de natureza quantitativa. O objetivo foi saber de que maneira se dá a eficiência da gestão da responsabilidade socioambiental nos hotéis-fazenda da Região Agreste de Pernambuco. Para a análise dos dados coletados foi utilizada a metodologia não paramétrica conhecida como a Análise Envoltória de Dados (DEA). O objetivo específico deste estudo foi mensurar a eficiência das ações de responsabilidade socioambiental a partir do uso da DEA.

\section{RESPONSABILIDADE SOCIAL EMPRESARIAL (RSE)}

A responsabilidade social empresarial não é um conceito novo, porém, tem 
despertado interesse entre acadêmicos e profissionais (HENDERSON, 2007). O tema tem sido palco de controvérsias sendo o seu conceito apresentado de forma difusa e, algumas vezes, contraditória. Expressões como cidadania empresarial, filantropia empresarial e responsabilidade social corporativa são empregadas como sinônimos, provocando dúvidas e abrindo lacunas a respeito de seu significado (REZENDE; JUNQUEIRA; MEDEIROS, 2008).

De acordo com Madrakhimova (2013), desde a década de 1950 que o campo da literatura científica e dos negócios, especialmente os EUA, teve como temática os assuntos relacionados com os problemas das empresas e da sociedade. Entretanto, a partir dos anos 80, predominaram os conceitos relacionados à ética empresarial, filantropia corporativa, política social corporativa e gestão de stakeholders. Já no começo do XXI, surgem teorias que envolvem o desenvolvimento sustentável, a cidadania corporativa, a sustentabilidade empresarial, a reputação corporativa e os investimentos socialmente responsáveis , relatórios sociais das empresas, entre outros (MADRAKHIMOVA, 2013).

Em 1971, de acordo com Carroll (1991) o Committee for Economic Development representava a RSE a partir do "Three Concentric Circles", o qual era apresentado graficamente da seguinte maneira: o círculo interno incluía funções básicas econômicas, crescimento, produtos e empregos. O círculo intermediário sugeriu que funções econômicas deveriam ser exercidas com uma consciência sensível para as mudanças de valores sociais e para as suas prioridades. O círculo externo delineava as responsabilidades de forma que empresa se tornasse mais ativamente envolvida no melhoramento do ambiente social (CARROLL, 1991).

Porém, de forma evoluída, surge a pirâmide da responsabilidade social corporativa de Carroll (1991), a qual se caracteriza por uma representação gráfica de forma hierárquica das responsabilidades do movimento econômico e jurídico empresarial orientando-se socialmente de forma ética e filantrópica (MEEHAN; MEEHAN; RICHARDS, 2006; CARROLL, 1991). Apesar de ter sido uma pespectiva promissora sobre a temática da responsabilidade social, os autores Schwartz e Carroll (2003) detectaram problemas inerentes à representação visual da pirâmide em relação à hierarquização entre os domínios da responsabilidade social, o desenvolvimento incompleto dos domínios econômico, legal e ético, o uso de uma categoria filantrópica separada, estando classificada melhor dentro das responsabilidades ética e/ou econômica.

Posteriormente, a pirâmide foi substituida pelo diagrama de Venn, o qual Schwartz e Carroll (2003) intitulam de The Three-Domain Model of Corporate Social Responsibility. Essa representação atualiza o modelo anterior e apresenta uma noção mais contemporânea da responsabilidade social corporativa, em que são demonstradas as sobreposições entre os domínios (MEEHAN; MEEHAN; RICHARDS, 2006; MORTATI, 2009).

Ainda que tenha evoluído para a melhora do modelo anterior, a representação, o modelo dos domínios possui, também, restrições explicitadas pelos autores Schwartz e Carroll (2003). Pressupõe-se que os domínios de responsabilidade social corporativa são, de certa forma, distintos e abrangem todos os tipos de atividades. Quanto ao primeiro aspecto, não se identifica qualquer ação como "puramente econômica", "puramente legal" ou "puramente ética", o que limita a aplicação prática ou conceitual de certos segmentos do modelo (MORTATI, 2009).

Ainda de acordo com Mortati (2009) no segundo ponto não está totalmente claro se existem atividades corporativas que não tenham relação com pelo menos um desses domínios. Ademais, relacionada à inclusão da categoria filantrópica dentro das categorias ética e/ou econômica, está 
a natureza inerentemente e conflitante de princípios éticos variados, que pode causar sérias dificuldades nessa classificação (MORTATI, 2009).

Apesar das restrições, o modelo The Three-Domain Model of Corporate Social Responsibility, proposto por Schwartz e Carroll (2003), apresenta-se como o mais adequado para esta pesquisa por considerar as sobreposições entre os componentes da responsabilidade social corporativa, assim como foi incorporado no estudo de (MORTATI, 2009). Logo, uma proposta que considera a sobreposição de componentes não exclui a estrutura conceitual do lado auxiliar do gestor na integração dos quatro componentes da responsabilidade social com os stakeholders organizacionais (CARROLL, 1991).

É fundamental destacar a importância do comprometimento que os meios de hospedagens possuem quanto ao atendimento da expectativa dos clientes e das partes interessadas, referentes à qualidade dos serviços e produtos oferecidos e aos desempenhos sociocultural e ambiental do negócio (ABNT, 2012).

\subsection{Gestão ambiental hoteleira}

A fim de alcançar uma melhoria, os gerentes de hotéis e os operadores devem estar dispostos a agir de forma ambientalmente responsável. Também é necessário possuirem um conhecimento adequado às questões ambientais pertinentes, às suas atividades e recursos suficientes para implementar práticas ambientalmente saudáveis e sustentáveis (BOHDANOWICZ, 2006) .

O planejamento da gestão ambiental a longo prazo determina a estratégia ambiental de uma empresa. Essa estratégia pode ser diferente dependendo do setor, das características de cada organização e do seu impacto sobre a ambiente (GIL; JIMÉNEZ; LORENTE, 2001).

Para Valle (2000), a gestão ambiental consiste em um conjunto de medidas e métodos, bem definidos e apropriadamente aplicados, que objetivam a redução e controle dos impactos introduzidos por um empreendimento sobre o meio ambiente. Frente a isso, os hotéis estão antenados para a problemática ambiental e estão agindo na direção de uma atitude ambientalmente responsável. Essa tem sido uma maneira encontrada pelos hoteleiros para reduzir seus custos, criar uma boa imagem perante o cliente e estabelecer um diferencial competitivo diante da concorrência acirrada (SANTOS; SOUZA; BARBOSA, 2005).

Inúmeros estudos surgiram para retificar a importância da gestão ambiental no segmento da hotelaria, tais como: Sistema de Gestão Ambiental como Estratégia Empresarial (SILVA FILHO, 2008); Certificação Ambiental em Turismo e Hospitalidade: o progresso, processo e as perspectivas (FONT, 2002); Atitudes face à gestão ambiental realizada por um grupo de gerentes de hotéis em Edimburgo (KIRK, 1998); Gestão Ambiental: um estudo em hotéis vietnamitas (LE et al., 2006); A consciência ambiental, ação e desempenho no Guernsey setor de hospitalidade (STABLER; GOODALI, 1997); A gestão ambiental de um destino turístico: um factor de competitividade do turismo (MIHALIC, 2000); Práticas de gestão ambiental entre hotéis da região Greater Accra (MENSA, 2006); Níveis de qualidade e gestão ambiental na indústria hoteleira: sua influência conjunta sobre o desempenho da empresa (TARI et al., 2010); Pesquisa em gestão estratégica no indústria da hospitalidade (OLSEN; ROPER, 1998); Uma análise da gestão ambiental, organizacional contexto e o desempenho dos hotéis espanhóis (GIL; JIMÉNEZ; LORENTE, 2001).

Esses debates, relacionados à indústria hoteleira, acontecem porque o segmento envolve, em sua cadeia produtiva, inúmeros atores que são impactados por suas ações. Assim, como trabalhado por Gil, Jiménez e Lorente (2001) busca-se 
concentrar esta pesquisa no setor da hotelaria tanto para seu papel de destaque nos serviços turísticos, como também pelo seu impacto sobre o meio ambiente.

Diversas iniciativas têm-se desenvolvido com o propósito de promover o turismo sustentável. Entre elas, o desenvolvimento de normas que estabeleçam os requisitos mínimos para o turismo sustentável, aliadas a mecanismos de certificação, que se tem destacado como uma das tendências mais presentes no mercado internacional, despertando o maior engajamento das partes interessadas (ABNT, 2006).

No Brasil, destacam-se as normas do sistema de gestão da sustentabilidade, na qual são somadas os demais modelos de sistemas de gestão já estabelecidos como a NBR ISO 9001 (Sistema de Gestão da Qualidade), a NBR ISO 14001 (Sistema de Gestão Ambiental). Esses sistemas contribuem para uma normatização mais ampla e integrada.

A norma ABNT NBR 15401 foi desenvolvida no âmbito do Comitê Brasileiro de Turismo - ABNT/CB 54 e foi publicada, no final de outubro de 2006, pela Associação Brasileira de Normas Técnicas (ABNT). A referência utilizada, para sua elaboração, foi a norma desenvolvida pelo Instituto de Hospitalidade (IH), dentro do Programa de Certificação em Turismo Sustentável (PCTS) (IH, 2004). Contudo a instituição não visa apenas conter os impactos ambientais no meio natural, mas também busca os resultados que irão favorecer ao empreendimento, tais como: - contribuir ativamente para a conservação, a revitalização e a recuperação dos recursos naturais;

- buscar resultados econômicos com ética, contribuindo para a justiça social e a valorização das culturas locais;

- buscar a legitimidade política em termos de participação e transparência nos processos de decisão e representação comunitária;

- interagir com os integrantes da cadeia produtiva do turismo, de maneira a construir as condições operacionais para implementar sistemas de gestão da sustentabilidade do turismo com abrangências setorial e geográfica.

Entretanto Erdogan e Baris (2007) constataram que apenas dois hotéis de quatro estrelas, investigados em sua pesquisa, destinaram parte de seu orçamento por razões ambientais. Da mesma forma, a maioria dos hotéis $(76,9 \%)$ investigados não têm pessoal responsável pela proteção ambiental. Nesse sentido, apresentam-se de forma necessária para que as empresas hoteleiras apliquem a metodologia de indicadores de sustentabilidade para mensurarem suas políticas (indicadores de comprometimento): Gestão (indicam planos, programas, metas e monitoramento); desempenho (indicadores de performance) e Cumprimento Legal (avaliam o cumprimento de normas nas áreas de concorrência, consumidor, trabalhista, ambiental, entre outras), e, com isso, garantirem um melhor desempenho sustentável empresarial.

\section{INDICADORES DE SUSTENTABI- LIDADE EMPRESARIAL}

De acordo com Bellen (2005), há inúmeras ferramentas de mensuração do Desenvolvimento Sustentável, porém destacam-se a Barometer of Sustainability, Ecological Footprint Method e Dashboard of Sustainability. Cada indicador trabalha com um conceito específico sobre o desenvolvimento sustentável (RABELO; LIMA, 2007). A palavra em latim Indicare, a qual significa apontar, descobrir, entre outras variações, originou o termo indicador (HAMMOND et al., 1995).

O processo diretivo sobre um determinado objetivo pode ser comunicado ou informado pelos indicadores, a exemplo do desenvolvimento sustentável; porém, em alguns casos, pode ser apreendido como um recurso que deixa mais perceptível uma tendência ou 
fenômeno que não seja imediatamente detectável (HAMMOND et al., 1995). No Brasil, são 59 os indicadores trabalhados pelo Instituto Brasileiro de Geografia e Estatística (IBGE), porém começou sua publicação com 50, em 2002 (RABELO; LIMA, 2007). Para os autores, a escolha do melhor indicador, ou seja, aquele que se pretende avaliar, surge a partir da percepção do pesquisador e é único para cada objeto de aplicação dessa metodologia.

Dessa maneira, o objetivo dos indicadores é agregar e quantificar as informações de modo que uma significância fique mais aparente, maximizando as informações sobre os fenômenos complexos na tentativa de melhorar a comunicação (BELLEN, 2004). O indicador também cumpriu a função social de melhorar a comunicação, mas pode desempenhar um papel útil apenas quando a comunicação é bem-vinda, quando a tomada de decisão é sensível a informações sobre questões sociais ou sobre a eficácia das políticas atuais (HAMMOND et al., 1995).

Os índices Ecological Footprint Method, Dashboard of Sustainability e Barometer of Sustainability são apresentados como as ferramentas mais relevantes, no contexto internacional, para a avaliação da sustentabilidade (BELLEN, 2004). Entretanto, no âmbito empresarial, tanto os indicadores e índices nacionais quanto os internacionais são abordados em pesquisas científicas. Entre eles, evidencia-se a Dow Jones Sustainability Indexes (DJSI), Global Reporting Initiative (GRI), o Environmental Performance Indicators (EPI's) e o Índice de Sustentabilidade Empresarial (ISE). Nesta pesquisa, faz-se uma adaptação do ISE como ferramenta de captação de dados.

Apesar de serem frequentemente apresentados de forma estatística ou gráfica, os indicadores são distintos das estatísticas ou dados primários. E de fato, os indicadores e índices são agregados ao topo de uma pirâmide de informação, cujas bases são dados primários, derivados de monitoramento e análise de dados
(HAMMOND et al., 1995).

Diante do exposto, nesta pesquisa, optou-se por utilizar o método de índice, pois acredita-se ser o mais sistemático para a pesquisa em questão e, também de acordo com o embasamento teórico, encontra-se no topo da pirâmide. Adota-se o Índice de Sustentabilidade Empresarial (ISE) por ser o quarto índice desse tipo que existe no mundo e sinaliza uma tendência, na qual visa destacar as empresas que, segundo a avaliação da Bovespa, apresentam melhores desempenhos em termos de responsabilidade social e sustentabilidade econômica, social e ambiental (LINS; SILVA, 2009).

\section{PROCEDIMENTOS METODOLÓ- GICOS}

O estudo tem como tema Modelo para a análise da eficiência de hotéis-fazenda com base na sua sustentabilidade. O problema de pesquisa busca responder à seguinte questão: de que maneira se dá a eficiência da gestão da responsabilidade socioambiental nos hotéis-fazenda da Região Agreste de Pernambuco? A pesquisa caracteriza-se como exploratória, descritiva e de corte transversal. A natureza dos dados é quantitativa e foi realizada com o objetivo de obter uma melhor compreensão sobre quais as práticas dos hotéis-fazenda em relação à responsabilidade socioambiental. O universo da amostra são seis hotéis-fazenda, no qual se fez o uso da amostragem por conveniência a partir dos seguintes critérios de inclusão:

i. Os hotéis-fazenda a serem pesquisados devem fazer parte da Associação Pernambucana de Turismo Rural e Ecológico (APETURR).

ii. Localização em destino turístico consolidado, nesse caso a Região Agreste, e que atendessem o mínimo de 20 UHs.

Entretanto, para fins de exemplificação numérica com dados reais, demarcaram-se apenas quatro hotéis-fazenda para exemplificar o modelo de mensuração da 
eficiência em relação à sustentabilidade. Todos os hotéis serão caracterizados na pesquisa, porém serão analisados apenas os quatro delimitados. As variáveis escolhidas foram baseadas em dois critérios: a pesquisa bibliográfica através de artigos de referência na temática e os dados disponíveis na coleta de dados.

Os hotéis escolhidos para a pesquisa estão localizados nos municípios de Gravatá, Bezerros, Bonito, Garanhuns e Sairé. Sendo o mais próximo da capital, Recife, o município de Gravatá, localizado a $86,9 \mathrm{Km}$ e o mais distante o município de Garanhuns, localizado a 231,5 Km da capital pernambucana.

$\mathrm{O}$ instrumento de coleta de dados foi através de aplicação de questionários estruturados aos gestores dos hotéis pesquisados. O questionário foi adaptado do Índice de Sustentabilidade Empresarial do Bovespa (ISE), indicado para a hotelaria, e engloba os aspectos sociais, ambientais e econômicos de forma mais aprofundada a partir dos seguintes critérios: político, gestão, desempenho e cumprimento legal.

A análise dos dados foi realizada mediante estatística descritiva, através do uso do software estatístico Efficiency Measurement System. A abordagem não paramétrica através do DEA foi utilizada para medir a eficiência dos hotéis-fazenda em relação à responsabilidade socioambiental pelo que foi necessário aplicar o uso das equações apresentadas no tópico da apresentação da modelagem.

\section{MODELAGEM DO PROBLEMA}

A Análise por Envoltória de Dados (DEA) se caracteriza como uma ferramenta para medir a eficiência relativa das unidades, na qual a analogia é realizada com o melhor grau de eficiência observado, ao invés de comparação com um ideal inatingível (SANTANA, 2008). Charnes, Cooper e Rhodes (1978) explanam algumas características consideradas positivas em relação ao método DEA, são elas:
- Utilização de múltiplos inputs e outputs.

- Diferencia as unidades eficientes das ineficientes.

- Detecta deficiências que não são constatadas por outras técnicas.

- Concebe um score de eficiência relativas às outras unidades produtivas.

Esse conjunto de benefícios, balizado em torno das técnicas DEA, torna sua escolha como métodos selecionados nesta investigação para obter perímetros mais claros e sistemáticos para que os objetivos da pesquisa sejam alcançados. Neste estudo, a intenção é que um método mensure a eficiência da gestão da responsabilidade socioambiental nos hotéis pesquisados.

De maneira geral, a eficiência evidencia o quão uma unidade de produção (por exemplo, fábrica, hospital, hotel, casa) é capaz de usar seus recursos para causar os resultados desejados (BROWN; RAGSDALE, 2002). Ou seja, interpreta-se para esta pesquisa que a eficiência da responsabilidade socioambiental de uma empresa, neste caso, os hotéis-fazenda, se reporta ao uso apropriado dos recursos naturais na intenção de gerar e agregar valor ao melhor serviço com menos custos, garantido uma competitividade no setor.

Nesse sentido, a avaliação de desempenho é uma parte necessária para o controle da gestão. Não só pode ser utilizado como referência na tomada de decisão, mas também serve como de base para quaisquer melhorias. À vista disso, medir a eficiência de um determinado setor, torna-se um assunto de grande relevância para o mercado (HWANG; CHANG, 2003).

O DEA é uma técnica flexível que pode incorporar múltiplas variáveis de inputs e de outputs para produzir um único índice de eficiência. $\mathrm{Na}$ análise DEA, as empresas ideais ficam sobre uma fronteira multidimensional chamada "melhor prática", porque representa as unidades mais eficientes na amostra. $\mathrm{O}$ método também envolve todas as empresas menos eficientes. O score de eficiência de cada 
empresa varia entre zero (eficiência mínima) e um (a máxima eficiência) (ASSAF; JOSIASSEN; CVELBAR, 2012).

O método teve como propulsor o trabalho de Farrell (1957), difundido, posteriormente, pelos autores Charnes, Cooper e Rhodes (1978). O primeiro introduziu a análise envoltória de dados para descrever o que é uma abordagem de programação matemática para a construção de produção de fronteiras e da medição da eficiência de fronteiras desenvolvidos. Os últimos autores propuseram um modelo - CCR - (em homenagem a eles mesmos) que tinha uma orientação input, e assumiram constantes retornos à escala (CRS) (BARROS, 2005). Já os autores Banker, Charnes e Cooper (1984) apresentam a classificação estabelecendo variáveis dentro do modelo chamado
BCC (nome atribuído em homenagem a eles mesmos).

O aspecto não paramétrico não se alicerça em uma função especificada $a$ priori. A forma da fronteira do conjunto produtivo é determinada considerando que o conjunto de produção deve satisfazer determinadas propriedades. O método DEA está inserido na abordagem não paramétrica, no qual se emprega o método de programação matemática para estimar modelos de fronteiras de produção e obter os escores de eficiência (FARRELL, 1957; BANKER; CHARNES; COOPER, 1984; BARROS, 2005; SAMPAIO; MELO, 2008). De acordo com a representação da figura 1 abaixo:

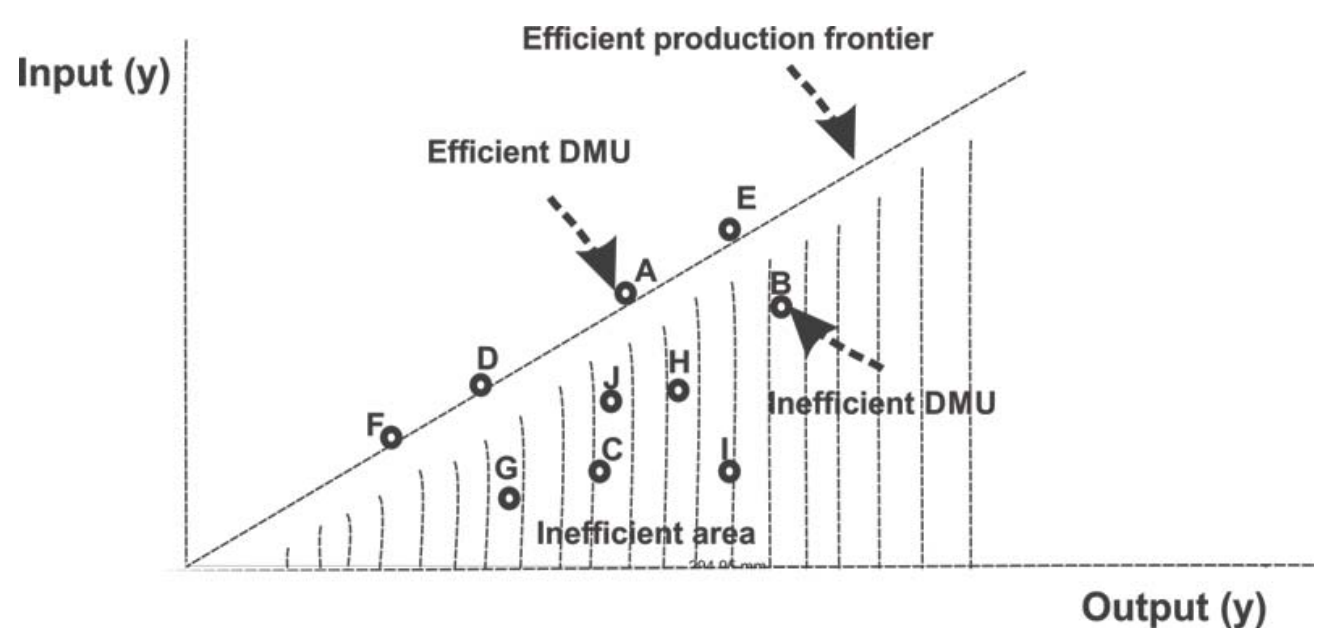

Figura 1 - Função para eficiência com base no DEA Fonte: Adaptado de Farrell (1957), Coell (1996) e Barros (2005)

Baseada na representação da eficiência técnica e distributiva orientada à entrada para uma unidade com duas saídas, estimou-se o gráfico representado na figura 1, onde se estipulou a fronteira de produção eficiente. Para representação de um hotel de forma simplista, tem-se que os hotéis A, D, E e F estão inseridos na curva de produção que define um hotel eficiente. Já os hotéis B, C G, H, I e J alocados abaixo da fronteira são categorizados como hotéis ineficientes. Sendo assim, os hotéis inseridos na curva de produção geram outputs, usando todas as entradas necessárias para seu funcionamento. Ou seja, se um dado hotel utiliza quantidades de entradas de forma ineficiente para produzir uma unidade de produto ou serviço, que é a quantidade pela qual todas as saídas podiam ser proporcionalmente aumentadas sem a necessidade de entradas adicionais (BARROS; MASCARENHAS, 2005). 
A eficiência $g 0$ da tomada de decisão da unidade (DMU), $j 0$, pode ser obtida resolvendo o valor máximo de uma proporção de saídas ponderadas nas entradas ponderadas sujeitas, na qual as

$\max h_{0}=\frac{\sum_{r=1}^{S} u_{r} y_{r 0}}{\sum_{i=1}^{M} v_{i} x_{i 0}}$

Subject 0 :

$\frac{\sum_{r=1}^{S} u_{r} y_{j}}{\sum_{i=1}^{M} u_{i} x_{j}} \leq 1 ; \quad j=1, \ldots, n ; \quad v_{r}, v_{i} \geq 0 ; \quad r=1, \ldots, s ; \quad i=1, \ldots, m$. proporções semelhantes para cada DMU seja inferior ou igual à unidade, de acordo com a proposta dos autores proposta abaixo (CHARNES; COOPER; RHODES, 1978):
O modelo apresentado pode ser resumido da seguinte maneira: $\mathrm{o} Y_{\mathrm{rij}^{\prime}} X_{\mathrm{ij}}$ são as saídas, as entradas são $j$ th $D M U$ e as $U_{\mathrm{r}^{\prime}}$ $V_{i} \geq 0$ são os pesos variáveis a serem determinados pela solução desse problema, por exemplo, com os dados sobre todo o DMU que estão a ser usados como um conjunto de referência. A eficiência do membro desse conjunto de referência de $j=1, \mathrm{n}$...., DMUs será avaliada em relação aos outros (CHARNES; COOPER; RHODES, 1978).

$\min f_{0}=\frac{\sum_{i=1}^{M} v_{i} x_{i 0}}{\sum_{r=1}^{S} u_{r} y_{r 0}}$

Subject o :

$$
\frac{\sum_{i=1}^{M} v_{i} x_{j}}{\sum_{r=1}^{S} u_{r} y_{j}} \geq 1 ; \quad j=1, \ldots, n ; \quad v_{i}, u_{r} \geq 0 .
$$

Após o modelo apresentado, os autores substituem os convexos não lineares das formulações com um problema de
Os autores sugerem, ainda, as reduções de formas na programação linear e propõem a utilização da teoria para fazer a formulação computacionalmente tratável para grande número de $j(\mathrm{n})$ de observações, bem como o menor número de entradas $i(\mathrm{~m})$ e saídas de $r(\mathrm{~s})$ que são prováveis que seja de interesse, pelo menos, nas aplicações econômicas como mostra a equação abaixo: programação linear ordinária. Portanto consideram o modelo: 
$\max z_{0}$

$-\sum_{j-1}^{n} y_{i j} \lambda_{j}+y_{r 0} z_{0} \leq 0 ; \quad r=1, \ldots, s$,

$\sum_{j=1}^{n} x_{j} \lambda_{j} \leq x_{i 0} ; \quad i=1, \ldots, m, \quad \lambda \geq 0 ; \quad j=1, \ldots, n$.

Seguidamente, os autores apresentam a estrutura da equação 4 , pode-se reconhecer que é equivalente a uma

$\min g_{0}=\sum_{i=1}^{m} w_{i} x_{i 0}$

Subject 0 :

$-\sum_{r=1}^{S} \mu_{r} y_{\dot{j}}+\sum_{i=1}^{m} w_{i} x_{j} \geq 0$

$\sum_{r=1}^{S} \mu_{r} Y_{r 0}=1, \quad \mu_{i} \geq 0$

Em suma, para resolver o problema de programação linear, o utilizador deve especificar três características do modelo: a orientação do sistema input-output, o retorno-à-escala e os pesos do sistema de avaliação. Em relação ao primeiro, a escolha de entrada ou saída orientada é baseada nas condições de mercado, na tomada de decisão da unidade. Regra geral, em mercados competitivos, as DMU são um output orientado, sendo que se presume estarem as entradas sob o controle do processo de unidade de decisão, que tem como objetivo maximizar a sua produção, em função da procura do mercado, fora do controle do DMU (BARROS, 2005). fração linear ordinária com problema de programação.

\subsection{Mensuração dos inputs e outputs}

Para o sucesso da aplicação de DEA, faz-se necessária uma cuidadosa identificação de inputs e outputs (YEN; OTHMAN, 2011). Aqui, neste trabalho, são estabelecidos os seguintes inputs: número total de funcionários, porcentagem de funcionários da localidade, número de leitos, consumo médio de energia, consumo médio da água e reúso médio da água, incorporação dos requisitos ambientais nos produtos e serviços. Os outputs são caracterizados por valor da diária com taxas, porcentagem da taxa de ocupação média, índices de satisfação dos clientes, porcentagem de resultados financeiros decorrentes de ações sustentáveis. De acordo com a tabela abaixo: 
Tabela 1 - Variáveis dos Inputs e Outputs para a avaliação da eficiência dos hotéis-fazenda.

\begin{tabular}{|l|}
\hline Inputs \\
\hline Número total de funcionários \\
\hline Porcentagem de funcionários da localidade \\
\hline Número de leitos \\
\hline Consumo médio de energia \\
\hline Consumo médio da água \\
\hline Reúso médio da água \\
\hline Incorporação dos requisitos ambientais nos produtos e serviços \\
\hline Outputs \\
\hline Valor da diária com taxas \\
\hline Porcentagem da taxa de ocupação média \\
\hline Índices de satisfação dos clientes \\
\hline Porcentagem de resultados financeiros decorrentes de ações sustentáveis \\
\hline
\end{tabular}

Fonte: Adaptado (KOKSAL; AKSU, 2007)

O output é uma medida consolidada de que uma organização atingiu seus objetivos. Como tal, se uma organização estabeleceu seus objetivos organizacionais, poderia estabelecer, imediatamente, critérios de avaliação e selecionar os fatores de insumo-produto. Na prática, os fatores de inputs e outputs são determinados pela experiência na formulação e implementação de planos operacionais, bem como pela disponibilidade de dados (HWANG; CHANG, 2003).

As variáveis categóricas selecionadas para mensurar a eficiência dos hotéis-fazenda pesquisados foram baseadas na literatura e na disponibilidade dos dados. Os seguintes estudos foram importantes para o estabelecimento dos inputs e outputs: Barros (2005), Hwang e Chang (2003), Koksal e Aksu (2007), Barros e Mascarenhas (2005), Assaf e Agbola (2014), Assaf, Deery e Jago (2011), Fernández e Becerra (2013) e Sampaio e Melo (2008).

\section{ANÁLISE}

O número de hotéis-fazenda da Região Agreste não é expressivo e são bastante homogéneos entre si garantindo um bom tratamento estatístico dos dados, por isso, o DEA foi o melhor método para este estudo. Dessa forma, as informações serão descritas estatisticamente e analisadas de maneira a explicitar a amplitude dos dados e, portanto, a homogeneidade existente.

Quanto ao tempo de funcionamento, dois hotéis têm mais de 20 anos, ou seja, já estão bem estabelecidos na região, sendo que um tem 12 anos, dois hotéis-fazenda têm 9 anos de funcionamento, e o restante (um hotel-fazenda) tem 5 anos de existência, indicando os vários estágios de consolidação dos hotéis-fazenda na região. Os municípios de Gravatá, Bezerros, Bonito e Garanhuns, onde estão os seis hotéis-fazenda pesquisados, são municípios que têm uma experiência turística no estado de Pernambuco, especialmente turismo interiorano com privilégio para regiões de clima ameno e belezas naturais no entorno. O hotel-fazenda localizado em Sairé está fora do circuito tradicional de turismo, porém observa-se que tem atributos locais (dentro da propriedade) de relevância e de cuidado com a valorização da vida no interior.

Observa-se, na amostra, um hotel-fazenda de grande fluxo com 220 funcionários e 88 apartamentos, outro com 48 funcionários e 60 apartamentos, outro 
com 22 funcionários e 74 apartamentos e, também, hotéis-fazenda estilo pousada com 14 funcionários e 22 aposentos. Estes configuram os extremos de observação. Os outros têm em média 28 funcionários e 23 apartamentos. As médias, desconsiderando esses extremos, ficam mais adequadas, pois essas amplitudes distorceriam a realidade optando-se, por isso, por retirar os que estão na maior e menor amplitude. Eles são administrados pelos proprietários e caracterizados como empresa familiar, possuindo de dois a seis proprietários. Todos situados no Agreste Setentrional de Pernambucano.

Apesar de se descrever sobre todo o universo, apenas serão exemplificados para fins de análise, os hotéis-fazenda HF1, HF2, HF3 e HF4. Os scores de eficiência provindos da DEA para a amostra de quatro hotéis-fazenda foram obtidos utilizando a seguinte execução do modelo: estrutura convexa, retorno de escala variável, distância radial e orientado via input. $\mathrm{Na}$ tabela 2 são apresentados os resultados com todos os inputs e outputs.

Tabela 2 - Score de eficiência hotéis-fazenda

\begin{tabular}{|l|c|c|c|c|}
\hline DMU & HF1 & HF2 & HF3 & HF4 \\
\hline SCORE & $100,00 \%$ & $100,00 \%$ & $100,00 \%$ & $98,91 \%$ \\
\hline FUNC $\{\mathrm{I}\}\{\mathrm{V}\}$ & 0,14 & 0,43 & 0,03 & 0 \\
\hline FUNCLOC $\{\mathrm{I}\}\{\mathrm{V}\}$ & 0,19 & 0,03 & 0,05 & 0,92 \\
\hline LEITO $\{\mathrm{I}\}\{\mathrm{V}\}$ & 0,09 & 0,15 & 0,07 & 0 \\
\hline ENERGIA $\{\mathrm{I}\}\{\mathrm{V}\}$ & 0,1 & 0 & 0,02 & 0 \\
\hline AGUA $\{\mathrm{I}\}\{\mathrm{V}\}$ & 0,1 & 0 & 0,75 & 0,01 \\
\hline REUSO $\{\mathrm{I}\}\{\mathrm{V}\}$ & 0,16 & 0,22 & 0,03 & 0 \\
\hline AMBIENTAL $\{\mathrm{I}\}\{\mathrm{V}\}$ & 0,22 & 0,16 & 0,04 & 0,07 \\
\hline TAXAS $\{\mathrm{O}\}\{\mathrm{V}\}$ & 0 & 0 & 0 & 0,74 \\
\hline OCUPACAO $\{\mathrm{O}\}\{\mathrm{V}\}$ & 0 & 1 & 0 & 0,03 \\
\hline SATIF $\{\mathrm{O}\}\{\mathrm{V}\}$ & 0 & 0 & 1 & 0,23 \\
\hline FINANC $\{\mathrm{O}\}\{\mathrm{V}\}$ & 1 & 0 & 0 & 0 \\
\hline & & & & $1(0,08)$ \\
BENCH & 1 & 1 & 1 & $2(0,90)$ \\
& & & & $3(0,02)$ \\
\hline
\end{tabular}

Fonte: Dados da pesquisa

O resultado gerado pela análise de DEA não indica somente os escores de eficiência das DMUs, mas apresenta, também, a frequência das unidades na qual possuem o melhor desempenho (BARROS; MASCARENHAS, 2005). Nesse caso, três de quatro hotéis-fazenda foram classificados como hotéis inseridos na fronteira de eficiência em relação à sustentabilidade (100\%). Porém apenas o hotel-fazenda HF4 mostrou-se abaixo da fronteira de eficiência com $(98,91 \%)$ comparado aos demais. $O$ resultado pode ser ocasionado por ser o hotel que possui o maior número de leitos $X$ taxa de ocupação e número médio de funcionários. Em relação à avaliação comparativa benchmarking, o hotel HF2 explicita-se como o exemplo a ser seguido pelos demais hotéis em análise. A tabela 3 apresenta alteração na eficiência. 
Tabela 3 - Eficiência dos hotéis-fazenda com inputs e outputs alterados

\begin{tabular}{|l|c|c|c|c|}
\hline \multirow{2}{*}{ DMU } & HF1 & HF2 & HF3 & HF4 \\
\hline SCORE & $80,00 \%$ & $100,00 \%$ & $100,00 \%$ & $63,01 \%$ \\
\hline FUNC $\{$ I $\}$ & 0 & 0,17 & 0,24 & 0,87 \\
\hline LEITO $\{$ I $\}$ & 1 & 0,03 & 0,01 & 0 \\
\hline ENERG $\{$ I $\}$ & 0 & 0,8 & 0,59 & 0,13 \\
\hline ÁGUA $\{I\}$ & 0 & 0 & 0,16 & 0 \\
\hline OCUP\{O\} & 1 & 1 & 0 & 0,26 \\
\hline SATISFAC $\{\mathrm{O}\}$ & 0 & 0 & 1 & 0,74 \\
\hline \multirow{2}{*}{ BENCH } & $2(0,60)$ & 2 & 2 & $2(0,93)$ \\
& $3(0,40)$ & $2(0,07)$ \\
\hline
\end{tabular}

Fonte: Dados da pesquisa

Entretanto o cenário modifica-se quando retiramos, para fins de confirmação da análise, os inputs: porcentagem de funcionários da localidade, reúso médio da água e incorporação dos requisitos ambientais nos produtos e serviços. Outputs: valor da diária com taxas e resultados financeiros decorrentes de ações sustentáveis. Os hotéis-fazenda HF3 e HF4 apresentam-se eficientes em relação à sus- tentabilidade sob os principais aspectos ambientais (água e energia) e operacionais (funcionários). No entanto, o HF1, quando retirado o fator econômico representado pelo output (valor da diária com taxas), exibe-se como ineficiente, assim como o HF4, que, mesmo com alteração dos inputs e outputs, não exibe indicadores suficientes para elevar-se ao status de eficiente no âmbito da sustentabilidade.

Tabela 4 - Eficiência dos hotéis-fazenda com inputs e outputs alterados

\begin{tabular}{|l|l|l|l|l|}
\hline DMU & HF1 & HF2 & HF3 & HF4 \\
\hline SCORE & $100,00 \%$ & $100,00 \%$ & $100,00 \%$ & $91,80 \%$ \\
\hline LEITO $\{$ I $\}$ & 0,12 & 0,75 & 0,07 & 0 \\
\hline AGUA $\{$ I $\}$ & 0,15 & 0 & 0,53 & 0,03 \\
\hline REUSO $\{\mathrm{I}\}$ & 0,27 & 0,22 & 0,28 & 0 \\
\hline AMBIENTAL $\{\mathrm{I}\}$ & 0,46 & 0,03 & 0,11 & 0,97 \\
\hline TAXAS $\{\mathrm{O}\}$ & 0 & 0 & 0 & 1 \\
\hline OCUP $\{\mathrm{O}\}$ & 0 & 1 & 0 & 0 \\
\hline SATISFAC $\{\mathrm{O}\}$ & 0 & 0 & 1 & 0 \\
\hline FINANC $\{\mathrm{O}\}$ & 1 & 0 & 0 & $1(0,03)$ \\
\hline & & 1 & & $2(0,83)$ \\
BENCH & 1 & & & $3(0,14)$ \\
\hline
\end{tabular}

Fonte: Dados da pesquisa 
Ao retirar os inputs de aspectos sociais do tripé da sustentabilidade (número total de funcionários e porcentagem de funcionários da localidade) e ambiental (consumo médio de energia), diminui ainda mais a discrepância de eficiência do HF5 (91,80\%) em relação aos demais. No que diz respeito à incorporação dos requisitos ambientais nos produtos $\mathrm{e}$ serviços, o HF1 possui destaque entre os eficientes. Todavia pressupõe-se que os domínios de responsabilidade social corporativa são, de certa forma, distintos e abrangem todos os tipos de atividades. Não se identifica qualquer ação como "puramente econômica", "puramente legal" ou "puramente ética" (MORTATI, 2009).

\section{CONCLUSÕES}

De um modo geral, os resultados globais mostram um grau de eficiência sustentável com discrepância de 2,8\% entre o hotel-fazenda inserido na fronteira de eficiência e não inserido. Apesar dessa rejeição, em relação ao HF4 é bastante expressivo o aspecto econômico com (74\%). Perante isto, conclui-se que os hotéis que, em alguma correlação, se alocaram como ineficientes devem observar claramente sua alocação na fronteira, pois, caso seja abaixo, devem ter o poder de decidir qual input ou output pode influenciar positivamente na realocação eficiente desses hotéis.

Também há a necessidade de se usar a DEA com cautela, pois a inclusão ou exclusão de variáveis (inputs e outputs) pode alterar completamente as eficiências relativas das unidades. Neste trabalho, por exemplo, o hotel HF1 mostrou-se ineficiente em relação ao aspecto da sustentabilidade quando se retirou osinputs: porcentagem de funcionários da localidade, reúso médio da água e incorporação dos requisitos ambientais nos produtos e serviços. Outputs: valor da diária com taxas e resultados financeiros decorrentes de ações sustentáveis, retificando a necessidade de se utilizar de forma adequada a modelagem.

Quando utilizados todos os inputs e outputs pré-definidos, três dos quatro hotéis-fazenda foram classificados como hotéis inseridos na fronteira de eficiência em relação à sustentabilidade (100\%). Porém apenas o hotel-fazenda HF4 mostrou-se abaixo da fronteira de eficiência, com $98,91 \%$, comparado aos demais.

Este artigo possui, como limitação principal, o número de DMUs limitado a quatro hotéis-fazenda, reduzindo a possibilidade de se generalizar o resultado. Sugere-se uma extensão desta pesquisa com a aplicação dessa mesma ótica de análise, porém em uma quantidade maior de hotéis-fazenda.

\section{REFERÊNCIAS}

ASSOCIAÇÃO BRASILEIRA DE NORMAS TÉCNICAS (ABNT). NBR 15401: Meios de Hospedagem - Sistema de Gestão da Sustentabilidade - Requisitos. Rio de Janeiro, 2006.

ASSAF, A. G.; DEERY, M.;JAGO, L. Evaluating the performance and scale characteristics of the australian restaurant industry. Journal of Hospitality E Tourism Research, v. 35, n. 4, p. 419-436, nov. 2011.

ASSAF, A. G.; JOSIASSEN, A.; CVELBAR, L. $K$. Does triple bottom line reporting improve hotel performance? International Journal of Hospitality Management, v. 31, n. 2, p. 596-600, jun. 2012.

ASSAF, A.; AGBOLA, F. W. Efficiency analysis of the australian accommodation industry: a bayesian output distance function. Journal of Hospitality \& Tourism Research, v. 38, n. 1, p. 116-132, fev. 2014.

BANKER, R. D.; CHARNES, A.; COOPER, $W$. W. Some models for estimating technical and scale inefficiencies in data envelopment analysis. Management Science, v. 30, n. 9, p. 1078-1092, set. 1984.

BARROS, C. P. Measuring efficiency in the hotel sector. Annals of Tourism Research, v. 32, n. 2, p. 456-477, abr. 2005. 
BARROS, C. P.; MASCARENHAS, M. J. Technical and allocative efficiency in a chain of small hotels. International Journal of Hospitality Management, v. 24, n. 3, p. 415-436, set. 2005.

BELLEN, H. M. Desenvolvimento sustentável: uma descrição das principais ferramentas de avaliação. Ambiente $\mathcal{E}$ Sociedade, v. VII, n. 1, jan./jun. 2004.

. Indicadores de sustentabilidade: uma análise comparativa. Rio de Janeiro: FGV, 2005.

BOHDANOWICZ, P. Environmental awareness and initiatives in the Swedish and Polish hotel industries - survey results. International Journal of Hospitality Management, v. 24, n. 4, p. 662-682, dez. 2006.

BROWN, J. R.; RAGSDALE, C. T. The competitive market efficiency of Hotel Brands: an application of data envelopment analysis. Journal of Hospitality $\mathcal{E}$ Tourism Research, v. 26, n. 4, p. 332-360, nov. 2002.

CARROLL, A. B. The pyramid of corporate social responsibility: toward the moral management of organizational stakeholders. Business Horizons, v. 34, n. 4, p. 39-47, jul./ ago. 1991.

CHAN, E. S. Implementing environmental management systems in small-and medium-sized hotels: obstacles. Journal of Hospitality $\mathcal{E}$ Tourism Research, v. 35, n. 1, p. 3-23, fev. 2011.

CHARNES, A.; COOPER, W. W.; RHODES, E. Measuring the efficiency of decision making units. European Journal of Operational Research, v. 2, n. 6, p. 429-444, nov. 1978.

CHARNES, A.; COOPER, W. W.; RHODES, E. Measuring the efficiency of decision making units. European Journal of Operational Research, v. 2, n.6, p. 429-444, nov. 1978.

COELL, T. J. A guide to DEAP version 2.1: a data envelopment analysis (computer) program. Working Paper: Centre for Efficiency and productivity analysis. University of New England, Armidale, Australia, 49, 1996.

EGRI, C. et al. Managerial perspectives on corporate environmental and social responsibilities in 22 countries. Academy of Management Proceedings, p. C1-C6, ago. 2004.

ERDOGAN, N.; BARIS, E. Environmental protection programs and conservation practices of hotels in Ankara, Turkey. Tourism Management, v. 28, n. 2, p. 604-614, abr. 2007.

FARRELL, M. J. The measurement of productive efficiency. Journal of the Royal Statistical Society, v. 120, n. 3, p. 253-290, 1957.

FERNÁNDEZ, M. A.; BECERRA, R. An analysis of spanish hotel efficiency. Cornell Hospitality Quarterly, v. 56, n. 3, p. 248-257, dez. 2013.

FONT, X. Environmental certification in tourism and hospitality: progress, process and prospects. Tourism Management, v. 23, n. 3, p. 197-205, jun. 2002.

GIL, M.; JIMÉNEZ, J. B.; LORENTE, J. C. An analysis of environmental management,organizational context and performance of Spanish hotels. Omega, v. 29, n. 6, p. 457-471, dez. 2001.

HAMMOND, A.; ADRIAANSE, A.; RODENBURG,E.;BRYANT,D.;WOODWARD, R. Environmental indicators: a systematic approach to measuring and reporting on environmental policy performance in the context of sustainable development. Washington: Wolrd Resources Institute, 1995.

HENDERSON, J. C. Corporate social responsibility and tourism: hotel companies in Phuket, Thailand, after the Indian Ocean tsunami. International Journal of Hospitality Management, v. 26, n. 1, p. 228-239, mar. 2007.

HWANG, S.-N.; CHANG, T.-Y. Using data envelopment analysis to measure hotel managerial efficiency change in Taiwan. Tourism Management, v. 24, n. 4, p. 357-369, ago. 2003.

INSTITUTO DE HOSPITALIDADE (IH). NIH54 - Norma Nacional para Meios de Hospedagem Requisitos para a Sustentabilidade. Salvador, BA, 2004.

KIRK, D. Attitudes to environmental management held by a group of hotel managers in Edinburgh. International Journal of HospitalityManagement, v. 17, n. 1, p. 33-47, mar. 1998.

KOKSAL, C. D.; AKSU, A. A. Efficiency evaluation of A-group travel agencies with data envelopment analysis (DEA): a case study in the Antalya region, Turkey. Tourism Management, v. 28, n. 3, p. 830-834, jun. 2007. 
LE, Y.; HOLLENHORST, S.; HARRIS, C.; MCLAUGHLIN, W.;SHOOK, S. Enviromental management: a study of vietnamese hotels. Annals of Tourism Research, v. 33, n. 2, p. 545567, 2006.

LINS, L. D.; SILVA, R. N. S. Responsabilidade sócio-ambiental ou Greenwash: uma avaliação com base nos relatórios de sustentabilidade ambiental. Sociedade, Contabilidade e Gestão, Rio de Janeiro, v. 4, n. 1, p. 91-105, jan./jun. 2009.

LUND-THOMSEN, P. Towards a critical framework on corporate social and environmental responsibility in the south: the case of Pakistan. Development, p. v. 47, n. 3, p. 106-113, 2004.

LYNES, J. K.; ANDRACHUK, M. Motivations for corporate social and environmental responsibility: a case study of Scandinavian Airlines. Journal of International Management, n. 14, p. 377-390, 2008.

MADRAKHIMOVA, F. S. Evolution of the concept and definition of corporate social responsibility. Global Conference on Business and Finance Proceedings, v. 8, n. 2, p. 113-117, 2013.

MCINTOSH, M. Raising a ladder to the Moon: The Complexities of Corporate Social and Environmental Responsibility. Palgrave Macmillan, New York, v. 27, n. 1, 2003.

MEEHAN, J.; MEEHAN, K.; RICHARDS, A. Corporate social responsibility: the 3C-SR model. International Journal of Social Economics, v. 33, n. 5/6, p. 386-398, maio/jun. 2006.

MENSAH, I. Environmental management practices among hotels in the greater Accra region. International Journal of Hospitality Management, v. 25, n. 3, p. 414-431, set. 2006.

MENSAH, I.; MENSAH, R. D. Internacional tourist's enviromental attitude towards hotels in Accra. Tourismos: An Internacional Multidisciplinary Journal of Tourism, Grécia, v. 8, n. 2, p. 57-75, 2013.

MIHALIC, T. Environmental management of a tourist destination: a factor of tourism competitivenes. Tourism Management, v. 21, n. 1, p. 65-78, fev. 2000.

MORTATI, I. B. Relacionando o comportamento organizacional e a responsabilidade social corpora- tiva. 2009. Dissertação (Mestrado em Gestão Empresarial) - Fundação Getúlio Vargas, Rio de Janeiro, 2009.

OLSEN, M. D.; ROPER, A. Research in strategic management in the hospitality industry. International Journal of Hospitality Management, v. 17, n. 2, p. 111-124, jun. 1998.

RABELO, L. S.; LIMA, P. V. Indicadores de sustentabilidade: a possibilidade da mensuração do desenvolvimento sustentável. REDE - Revista Eletrônica do Prodema, Fortaleza, CE, v. 1, n. 1, p. 55-76, dez. 2007.

REZENDE, J. M.; JUNQUEIRA, M. C.; MEDEIROS, C. R. Responsabilidade social empresarial: uma prática de gestão. In: SEMANA DOSERVIDOR, 4., SEMANA ACADÊMICA, 5. Uberlância MG: Universidade Federal de Uberlândia, 2008. Disponível em: <https:// ss14799.websiteseguro.com/swge5/seg/ cd2008/PDF/SA08-20650.PDF>.

ROSEMBERG, W. Making a profit... and a difference: HP invents na organization to drive sustainability. Journal of Organizational Excellence, v. 23, n. 3, p. 3-13, 2004.

SAMPAIO, B.; MELO, A. S. Análise da eficiência de companhias aéreas brasileiras. Revista Análise Econômica, Porto Alegre, RS, v. 26, n. 50, p. 223-244, set. 2008.

SANTANA, N. B. Responsabilidade socioambiental e valor da empresa: uma análise por envoltória de dados em empresas distribuidoras de de energia elétrica. 2008. 328p. Dissertação (Mestrado em Engenharia de Produção) - Escola Engenharia de São Carlos, Universidade de São Paulo, São Carlos, SP, 2008.

SANTOS, C. B.; SOUZA, M. T.; BARBOSA, R. J. Gestão ambiental em empreendimentos hoteleiros: análise de práticas e de resultados em um estudo de casos múltiplos. In: SIMPÓSIO DE EXCELÊNCIA EM GESTÃO E TECNOLOGIA - SEGeT, 3. 2005. p. 1-12.

SCHAEFER, A. Corporate sustainability - integrating environmental and social concerns? Corporate Social Responsibility and Environmental Management, p. 179 -187, nov. 2004.

SCHWARTZ, M. S.; CARROLL, A. B. Corporate social responsibility: a three-domain approach. Business Ethics Quarterly, v. 13, n. 4, p. 503-530, out. 2003. 
SILVA FILHO, A. R. Sistema de gestão ambiental como estratégia empresarial no ramo hoteleiro. Produção Online - Revista Científica Eletrônica de Engenharia de Produção, v. 8, n. 3, p. 1676-1901, 2008.

SILVA, R. F. Turismo, desenvolvimento sustentável e direitos humanos: O programa "Viaja Mais, Melhor Idade". Revista Brasileira de Pesquisa em Turismo, v. 6, n. 3, p. 290-304, 2012.

STABLER, M. J.; GOODALI, B. Environmental awareness, action and performance in the Guernsey hospitality sector. Tourism Management, v. 18, n. 1, p. 19-33, 1997.

SUESS, C. Systematic analysis of identif ying key dimensions of environmentally and socially responsible hotels. 2009. Disserta- ção (Master of Hospitality Administration) - William F. Harrah College of Hotel Administration, Universidade de Nevada, Las Vegas, 2009.

TARI, J. J.; CORTES, E. C.; MOLINE, J. P.; AZORIN, J. F. Levels of quality and environmental management in the hotel industry: their joint influence on firm performance. International Journal of Hospitality Management, v. 29 , n. 3, p. 500-510, set. 2010.

VALLE, C. E. Como se preparar para as normas ISO 14000: qualidade ambiental.São Paulo: Pioneira, 2000.

YEN, F. L.; OTHMAN, M. Data envelopment analysis to measure efficiency of hotels in Malaysia. SEGi Review, v. 4, n. 1, p. 25-36, jul. 2011.

\section{Sobre os autores:}

Viviane Souza: Doutoranda em Turismo pela Universidade de Aveiro. Mestre em Administração pela Universidade Federal Rural de Pernambuco. E-mail: vivianne. turismologa@gmail.com.br

Andre de Souza Melo: Doutor em Economia pela UFPE. Professor do Programa de Pós-Graduação em Administração da Universidade Federal Rural de Pernambuco. E-mail: andredesouzam@gmail.com

Marcos Felipe Falcão Sobral: Doutor em Engenharia de Produção. Professor Adjunto do Programa de Pós-Graduação em Administração e Desenvolvimento na UFRPE. E-mail: marcos_sobral@bol.com.br

Marília Nunes Valença: Bacharel em Turismo pela UFPE. Mestranda em Administração pela Universidade Federal Rural de Pernambuco. E-mail: marilia_nv@hotmail.com 
